\title{
Discrete Laplace-Beltrami Operators for Shape Analysis and Segmentation
}

\author{
Martin Reuter ${ }^{\mathrm{a}, \mathrm{b}}$, Silvia Biasottic ${ }^{\mathrm{c}}$, Daniela Giorgic ${ }^{\mathrm{c}}$, Giuseppe Patanèc ${ }^{\mathrm{c}}$, Michela Spagnuolo $^{\mathrm{c}}$ \\ ${ }^{a}$ Massachusetts Institute of Technology, Cambridge, MA, USA \\ ${ }^{b}$ A.A. Martinos Center for Biomedical Imaging, Massachusetts General Hospital, Harvard Medical School, Boston, MA, USA \\ ${ }^{c}$ Istituto di Matematica Applicata e Tecnologie Informatiche - Consiglio Nazionale delle Ricerche, Genova, Italy
}

\begin{abstract}
Shape analysis plays a pivotal role in a large number of applications, ranging from traditional geometry processing to more recent 3D content management. In this scenario, spectral methods are extremely promising as they provide a natural library of tools for shape analysis, intrinsically defined by the shape itself. In particular, the eigenfunctions of the Laplace-Beltrami operator yield a set of real valued functions that provide interesting insights in the structure and morphology of the shape. In this paper, we first analyze different discretizations of the Laplace-Beltrami operator (geometric Laplacians, linear and cubic FEM operators) in terms of the correctness of their eigenfunctions with respect to the continuous case. We then present the family of segmentations induced by the nodal sets of the eigenfunctions, discussing its meaningfulness for shape understanding.
\end{abstract}

Key words: Laplace-Beltrami operator, eigenfunctions, nodal sets, nodal domains, shape analysis, shape segmentation.

\section{Introduction}

Shape analysis aims to develop computational tools for reasoning on properties of the objects' shape, and is pivotal in a large number of applications, ranging from traditional geometry processing to more recent 3D content management techniques.

In the recent past, research in shape analysis was boosted by the need to add semantics to the geometric description of 3D objects, in order to facilitate the sharing and management of 3D content in many emerging web-based applications. A semantic description of 3D objects is commonly understood as a description of the content by means of terms which are meaningful in some domain of knowledge. For example, a given model can be described as being a table, made of four cylindrical legs and an oval top. Hence, a semantic description calls for segmentation algorithms which capture semantically relevant features in an automatic manner.

Most of the methods developed so far for shape analysis and segmentation do not directly provide any semantically-relevant explicit description of the shape, but rather provide a characterization of the geometric and structural properties of the object boundary. Semantic properties are taken into account, at some extent, by cognitive theories supporting part-based decompositions or minima rule-based approaches. Part-based decomposition techniques build on Biederman's theory of perception, which characterizes an object as a compound of primitive basic parts (e.g., planes, spheres, cylinders, cubes) [10]. The second class of methods are based on the so-called minima rule, which suggests that we perceive relevant parts by focusing

Email addresses: reuter@mit .edu (Martin Reuter), silvia@ge.imati.cnr.it (Silvia Biasotti), daniela@ge.imati.cnr.it (Daniela Giorgi), patane@ge.imati.cnr.it (Giuseppe Patanè), michi@ge.imati.cnr.it (Michela Spagnuolo) our attention on lines of concave discontinuity of the tangent plane [26]. For a recent survey of segmentation methods, we refer the reader to [54].

Our interest is in the development of methods for shape analysis and segmentation able to capture a varied set of morphologically relevant features, possibly at different scales: in other words, we seek for a library of tools supporting the semantic annotation of digital shapes. Shape understanding, indeed, is a very complex task and it is now widely accepted that no single segmentation method is capable of capturing relevant features in a broad domain of shapes. In [3], shape understanding is seen as a multi-segmentation task driven by the user, who uses in parallel a set of segmentation algorithms and composes the final segmentation with selection and refinement operations on the segments. In that work, the authors push forward the idea of semantic annotation by allowing the user to associate textual tags, defined in an ontology, to the segments.

In this scenario, spectral methods are extremely promising, as they naturally provide a set of tools for shape analysis that are intrinsically defined by the shape itself. Spectral methods have recently gained much interest in computer graphics [62], with applications that include mesh compression [30], parametrization [25, 43], segmentation [31, 35], remeshing [17], filtering [34, 57], correspondence [27], matching and retrieval [28, 49, 52], manifold learning [5], and imaging or medical imaging applications $[44,38,50]$.

In particular, the eigenfunctions of the Laplace-Beltrami operator yield a family of real valued functions that provide interesting insights in the structure and morphology of shapes. In this paper, we focus on the nodal sets of the Laplace-Beltrami eigenfunctions, showing that they induce a shape decomposition which captures features at different scales, generally wellaligned with perceptually relevant shape features. The set of 
decompositions induced by the eigenfunctions yields the sought library of intrinsic shape segmentations.

The first contribution of this paper is the analysis of the correctness of the eigenfunctions computed using different discretizations of the Laplace-Beltrami operator (Section 2), evaluated with respect to the exact results known from the theory in the continuous case (Section 3 ). The second contribution is the introduction of the set of segmentations induced by the nodal sets of the eigenfunctions; the segmentations are discussed in terms of their quality and robustness (Section 4). Finally, we draw some conclusive remarks and highlight possible extensions of this work (Section 5).

\section{The Laplace-Beltrami operator}

Let $f$ be a $C^{2}$ real-valued function defined on a differentiable manifold $\mathcal{M}$ with Riemannian metric [7]. The LaplaceBeltrami operator $\Delta$ is

$$
\Delta f:=\operatorname{div}(\operatorname{grad} f)
$$

where grad and div are the gradient and divergence on the manifold $\mathcal{M}$ [11]. The Laplacian eigenvalue problem is stated as

$$
\Delta f=-\lambda f
$$

Since the Laplace-Beltrami operator is self-adjoint and semipositive definite [51], it admits an orthonormal eigensystem $\mathcal{B}:=\left\{\left(\lambda_{i}, \psi_{i}\right)\right\}_{i}$, that is a basis of the space of square integrable function, with $\Delta \psi_{i}=\lambda_{i} \psi_{i}, \lambda_{0} \leq \lambda_{1} \leq \ldots, \lambda_{i} \leq \lambda_{i+1} \ldots \leq+\infty$. For a detailed discussion on the main properties of the LaplaceBeltrami operator, we refer the reader to $[46,51,59]$.

\subsection{The discrete case}

The solution to (1) on a surface is frequently approximated by a piecewise linear function $f: \mathcal{T} \rightarrow \mathbb{R}$ over a triangulation $\mathcal{T}$ with vertices $V:=\left\{\mathbf{p}_{i}, i=1, \ldots, n\right\}$. The function $f$ on $\mathcal{T}$ is defined by linearly interpolating the values $f\left(\mathbf{p}_{i}\right)$ of $f$ at the vertices of $\mathcal{T}$. This is done by choosing a base of piecewiselinear hat-functions $\varphi_{i}$, with value 1 at vertex $\mathbf{p}_{i}$ and 0 at all the other vertices. Then $f$ is given as $f=\sum_{i=1}^{n} f\left(\mathbf{p}_{i}\right) \varphi_{i}$. Discrete Laplace-Beltrami operators are usually represented as

$$
\Delta f\left(\mathbf{p}_{i}\right):=\frac{1}{d_{i}} \sum_{j \in N(i)} w_{i j}\left[f\left(\mathbf{p}_{i}\right)-f\left(\mathbf{p}_{j}\right)\right],
$$

where $N(i)$ denotes the index set of the 1-ring of the vertex $\mathbf{p}_{i}$, i.e. the indices of all neighbors connected to $\mathbf{p}_{i}$ by an edge. The masses $d_{i}$ are associated to a vertex $i$ and the $w_{i j}$ are the symmetric edge weights. To write (2) in matrix form, we define the vector $\mathbf{f}:=\left(f\left(\mathbf{p}_{1}\right), \ldots, f\left(\mathbf{p}_{n}\right)\right)^{T}$ of the function values at the vertices, the weighted adjacency matrix $W:=\left(w_{i j}\right)$, and the diagonal matrix $V:=\operatorname{diag}\left(v_{1}, \ldots, v_{n}\right)$ containing as diagonal elements $v_{i}=\sum_{j \in N(i)} w_{i j}$. Then, we can define a stiffness matrix $A:=V-W$, the lumped mass matrix $D:=\operatorname{diag}\left(d_{1}, \ldots, d_{n}\right)$, and finally the Laplace matrix $L:=D^{-1} A$ (generally not symmetric). Using these matrices, $\Delta f\left(\mathbf{p}_{i}\right)$ is the $i$-th component of the vector $L \mathbf{f}$. The problem (1) can then be written as $L \mathbf{f}=\lambda \mathbf{f}$ or better as a generalized symmetric problem $A \mathbf{f}=\lambda D \mathbf{f}$. In the following, we distinguish between geometric operators and finite-element operators on the basis of different edge weights and masses.

Discrete geometric Laplacians. A very simple choice of weights $w_{i j}$ for a graph is the adjacency matrix ( 1 if $\mathbf{p}_{i}$ and $\mathbf{p}_{j}$ are connected by an edge, 0 otherwise) and unit masses $d_{i}=1$. This operator and simple variations are called graph Laplacians as they usually only consider the connectivity and no geometry. Lévy [33] gives a very good overview and compares this graph Laplacian with a discretization by Desbrun et al. [16] (presented below).

One of the early geometric approaches has been described by Pinkall and Polthier [45], who discretize the Laplace-Beltrami operator using constant masses (i.e., $d_{i}:=1$ ) in (2) and weights

$$
w_{i j}:=\frac{\cot \left(\alpha_{i j}\right)+\cot \left(\beta_{i j}\right)}{2},
$$

where $\alpha_{i j}$ and $\beta_{i j}$ denote the two angles opposite to the edge $(i, j)$. Because of the lack of a proper mass weighting the cotangent weights alone still depend on mesh sampling.

Desbrun et al. [16] refine the discretization in (3) by using a normalization factor, which takes into account the area $a(i)$ of all triangles at vertex $i$, i.e.

$$
d_{i}:=a(i) / 3
$$

Lévy [33] uses this operator but instead of solving the symmetric generalized problem $A \mathbf{f}=\lambda D \mathbf{f}$ he looks at the nonsymmetric matrix $L=D^{-1} A$ and then computes the eigenvalues and eigenfunctions of the symmetric matrix $\left(L+L^{t}\right) / 2$ which yields a different spectrum.

Meyer et al. [36] modify the area normalization by Desbrun and propose the mass weighting

$$
d_{i}:=a_{V}(i)
$$

with $a_{V}(i)$ the area obtained by joining the circumcenters of the triangles around vertex $i$ (i.e., the Voronoi region). Founding on discrete exterior calculus, $[15,34]$ reach the same operator. Lévy and Vallet [34] symmetrize the operator by using $1 / \sqrt{a_{V}(i) a_{V}(j)}$ instead of the inversion of the mass matrix $1 / a_{V}(i)$. This leads to the system $D^{-1 / 2} A D^{-1 / 2} \mathbf{y}=\lambda \mathbf{y}$ with the same eigenvalues. The original eigenvectors can be retrieved by $\mathbf{f}=D^{-1 / 2} \mathbf{y}$.

Belkin et al. [5, 6] describe a discretization of the LaplaceBeltrami operator on the $k$-nearest neighbor graph $\mathcal{T}$ of a point set $\left\{\mathbf{p}_{i}\right\}_{i=1}^{n}$ sampled on an underlying manifold and an extension to meshes by using the heat kernel to construct the weights. The mesh version [6] considers weights not only at the edges of the mesh, but in a larger neighborhood of a vertex (the heat kernel is cut off thus sparsity is maintained). While the geometric operators in $(3,4,5)$ are not convergent in general and cannot deal well with non-uniform meshes [60], this method exhibits convergence and does not depend much on the shape of the triangles, just on the density of the vertices. However, it can be 

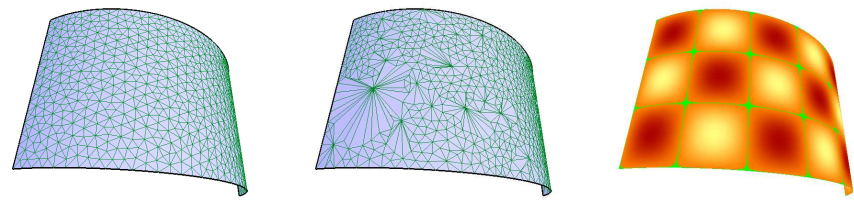

Figure 1: Curved rectangle uniform mesh, non-uniform mesh, real eigenfunction 24 ; the green lines denote the zero level sets.

used to compute eigenfunctions only on closed meshes, as it is unclear how to comply with the Dirichlet or Neumann boundary condition. Another discretization by Floater can be found in [22], but is not a good choice for eigencomputations due to its non-symmetry.

Discrete FEM Laplacians. The solution of the Laplace eigenvalue problem (1) can be computed by imposing that the equation $\Delta f=-\lambda f$ is verified in a weak sense, that is,

$$
\left\langle\Delta f, \varphi_{i}\right\rangle_{\mathcal{L}^{2}(\mathcal{M})}=-\lambda\left\langle f, \varphi_{i}\right\rangle_{\mathcal{L}^{2}(\mathcal{M})}, \quad \forall i .
$$

This was done first for triangle surface meshes with a linear finite element method (FEM) in [20], for parametrized surfaces, triangle and tetrahedra meshes using higher order FEM in [48, 49] and for voxel data in [47]. The discrete setting (6) with linear finite elements is equivalent to the generalized eigenvalue problem

$$
A_{\mathrm{cot}} \mathbf{f}=-\lambda B \mathbf{f}, \quad \mathbf{f}:=\left(f\left(\mathbf{p}_{i}\right)\right)_{i=1}^{n},
$$

where

$$
\begin{gathered}
A_{\cot }(i, j):= \begin{cases}\frac{\cot \alpha_{i j}+\cot \beta_{i j}}{2} & (i, j) \text { edge } \\
-\sum_{k \in N(i)} A_{\cot }(i, k) & i=j,\end{cases} \\
B(i, j):= \begin{cases}\frac{\left|t_{1}\right|+\left|t_{2}\right|}{12} & (i, j) \text { edge } \\
\frac{\sum_{k \in N(i)}\left|t_{k}\right|}{6} & i=j,\end{cases}
\end{gathered}
$$

$\left|t_{i}\right|$ is the area of the triangle $t_{i}$, and $t_{1}, t_{2}$ are the triangles that share the edge $(i, j)$, and $A_{\text {cot }}$ is the stiffness matrix with cotangent weights (see also [34, 58]). Note that by lumping the mass matrix $B$ (i.e., adding all entries per row to the diagonal element) one obtains the diagonal mass matrix $d_{i}:=a(i) / 3$ and therefore the same operator as Desbrun et al. [16]. See also [44] Section 4.3 for a discussion on the graph Laplacian and the connection to the FEM case on a regular grid.

The full FEM approach is superior to the discrete geometric Laplace operators. As opposed to the diagonal matrix $D$, the inner product $B=(B(i, j)):=\iint \varphi_{i} \varphi_{j} d \sigma$ correctly represents the discrete counterpart of the continuous inner product (for $f=\sum_{i=1}^{n} f\left(\mathbf{p}_{i}\right) \varphi_{i}$ and $g=\sum_{i=1}^{n} g\left(\mathbf{p}_{i}\right) \varphi_{i}$ the product is $\iint f g d \sigma=\mathbf{f}^{T} B \mathbf{g}$ ). Furthermore, the FEM approach is very general and can be extended easily to higher dimensions (e.g. tetrahedra or voxel meshes) and higher order computations by choosing a set $\mathcal{F}:=\left\{\varphi_{i}\right\}_{i}$ of higher order form functions while introducing new nodes within each triangle; for more details on the cubic finite elements, we refer the reader to $[49,46]$. FEM discretizations have strong convergence results. In fact, with
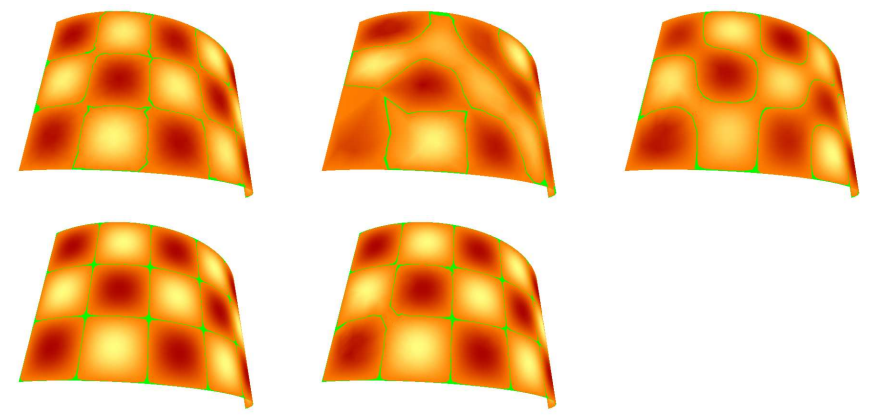

Figure 2: Approximated eigenfunction 24. Linear FEM (top row) and cubic FEM (bottom row) for the uniform (left), non-uniform (middle) and nonuniform refined mesh (right).

decreasing mesh size $h$ and order $p$ form functions the eigenvalues converge with order $2 p$ and the eigenfunctions with order $p+1$ in the $L_{2}$ norm [56] (as long as the geometry is represented correctly). Actually, this is the reason why one should always prefer higher order FEM approximations over a mesh refinement. Experimental evidence is given in Section 3.

Solution of the eigenvalue problem. For a generalized symmetric eigenvalue problem $A \mathbf{f}=\lambda B \mathbf{f}$ with $A$ positive semidefinite and $B$ positive definite, we can find non-negative eigenvalues $\lambda$ and eigenvectors $\mathbf{f}$ such that we always have [40]

$$
X^{T} A X=\Lambda \text { and } X^{T} B X=I .
$$

Here, $I$ is the identity, $\Lambda:=\operatorname{diag}\left(\lambda_{1}, \ldots, \lambda_{n}\right)$ is the diagonal matrix of the eigenvalues, and $X:=\left(\mathbf{f}_{1}, \mathbf{f}_{2}, \ldots, \mathbf{f}_{n}\right)$ is the matrix of the eigenvectors. From the previous relations, it follows that the eigenfunctions of the geometric and FEM Laplacian matrix are orthogonal with respect to the $B$-based scalar product (i.e., $\left.\langle\mathbf{x}, \mathbf{y}\rangle:=\mathbf{x}^{T} B \mathbf{y}\right)$.

Often only a small number of eigenvalues and eigenfunctions are needed. Once the matrices $A$ and $B$ (or $D$ ) are constructed, the generalized symmetric eigenvalue problem $A \mathbf{f}=\lambda B \mathbf{f}$ needs to be solved. Note that even for a diagonal matrix $D$, this is usually preferable and more stable than inverting $D$ or transforming the system to a similar system $D^{-\frac{1}{2}} A D^{-\frac{1}{2}} \mathbf{y}=\lambda \mathbf{y}$ with $\mathbf{y}:=D^{\frac{1}{2}} \mathbf{f}$. For the computation, we follow [48, 49]. As this is a large and sparse problem an iterative Krylov methods (such as Lanczos) can be used very efficiently. In this work, we use the shiftinvert method as implemented in ARPACK [1]. Because iterative methods work best on large eigenvalues, the problem is first inverted into $B \mathbf{f}=\frac{1}{\lambda} A \mathbf{f}$. Then, for the iterative algorithm only the product $\mathbf{z}=B \mathbf{f}$ needs to be computed and $A \mathbf{y}=\mathbf{z}$ needs to be solved repeatedly, which is done by computing a sparse LU decomposition of $A$ once. We use ARPACK for solving the shift-inverse problem and SuperLU [14] for the factorization. The function calls are managed through the $\mathrm{C}++$ wrapper Arpack++ [24].

\section{Correctness of the discretized operators}

Previous results on the accuracy of discrete operators [19, 49] have been mostly focused on the analysis of the behavior of the 

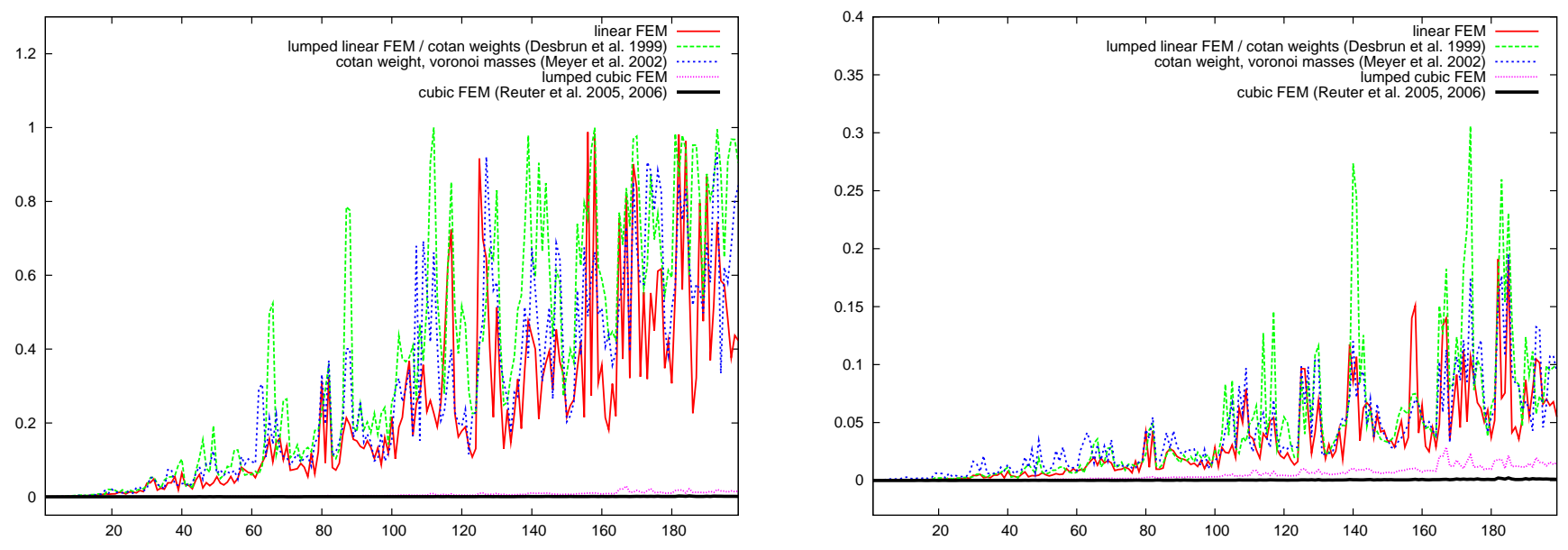

Figure 3: Left: Error of eigenfunctions on the flat rectangular domain. Right: Comparison with linear methods on the globally refined mesh.

computed eigenvalues with respect to changes of the mesh connectivity and sampling. In [19], the authors evaluate the robustness of the cotangent operator [45], lumped FEM discretization [16], and linear FEM discretization [49] by studying the change in the spectrum due to different mesh connectivity and sample density. It is claimed that looking at the eigenvalues instead of the eigenvectors is easier but still informative if one assumes that the robustness of eigenvalues implies robustness of eigenfunctions. Anyway, the authors only show that the opposite direction is true, i.e., good eigenfunctions imply good eigenvalues. Also, for higher order FEM it is easier to obtain good eigenvalues than functions as a consequence of the faster convergence of the eigenvalues with respect to the eigenfunctions. Finally, especially in cases where the results do not converge to the correct results, picking a fine mesh resolution is completely arbitrary and maybe has larger error than a coarse mesh result.

Hence, we take a different approach to better analyze the properties of the Laplacian eigenfunctions. To this end, we compare the computed eigenfunctions with the correct results known from theory, instead of comparing with a finer mesh resolution. Since the exact eigenfunctions are known for rectangles and spheres, we compute the approximated eigenfunctions using different discretizations of the Laplace-Beltrami operator and compare their correctness by evaluating the error with respect to the exact eigenfunctions. Based on the same principle, we can verify the effects of different samplings of models and isometric transformations.

More precisely, we have considered a rectangular domain with $a=1, b=2$ as side length, and generated various meshes with dense, coarse, uniform or irregular sampling density. We have also considered mapping the rectangle onto a cylinder shell, via the transformation $x \rightarrow \cos (x), y \rightarrow y, z \rightarrow \sin (x)$ that isometrically maps a straight segment of length $\pi$ into a half-circle with radius 1 . Then, we have compared the eigenfunctions computed on the meshes with the exact ones known from theory. For the rectangular domain with side length $a$ and $b$ and Neumann boundary condition, these are

$$
\psi_{m, n}=\cos \left(\frac{m \pi}{a} x\right) \cos \left(\frac{n \pi}{b} y\right) \quad m, n \in\{0,1,2, \ldots\} .
$$

In this case, the eigenvalues $\lambda_{m, n}=\pi^{2}\left(\frac{m^{2}}{a^{2}}+\frac{n^{2}}{b^{2}}\right)$ determine the order and the dimension of the eigenspaces [46].

Given the uniform and non-uniform mesh of the curved rectangle shown in Figure 1, Figure 2 shows a visual comparison of the 24th Dirichlet eigenfunction for the linear and cubic FEM case. It can be seen that for the uniform mesh the result of the cubic FEM (bottom left) is very close to the exact result in Figure 1 (right). For the non-uniform mesh the linear (top middle) performs very poorly, while the cubic (bottom middle) stays close to the exact result. This is expected as the cubic approach has more degrees of freedom (i.e., 10 nodes instead of 3 per triangle). Therefore, a fair comparison was done by globally refining the mesh (subdividing each triangle into 9 similar ones) so that the linear FEM on this mesh has the same degrees of freedom as the cubic case. It can be seen in Figure 2 (top right) that linear FEM still cannot reach the accuracy of cubic FEM.

To compare different discrete operators, we will analyze the first 200 Neumann eigenfunctions of a flat rectangular domain and of the sphere, but first we need to solve the following difficulties. Because in higher dimensional eigenspaces, any linear combination could be a solution, a direct comparison as shown above is possible only in case of 1-dimensional eigenspaces. Moreover, we have to take into account the sign ambiguity of eigenfunctions. To bypass these problems, we take a base of the eigenspace of the correct solutions (where the dimension is known). Then, we project the approximated results $\tilde{f}$ onto this space and compute the difference between this projection $P(\tilde{f})$ and the approximated eigenfunction $\tilde{f}$. If the latter lies within this space, the difference will be zero, the projection will be the function itself. If $\tilde{f}$ lies outside the space, we get a larger difference; i.e., up to 1 if the function is orthogonal to the eigenspace. The advantage of this approach is that it can be applied to both higher dimensional and 1-dimensional eigenspaces, also solving the problem of sign ambiguity. 

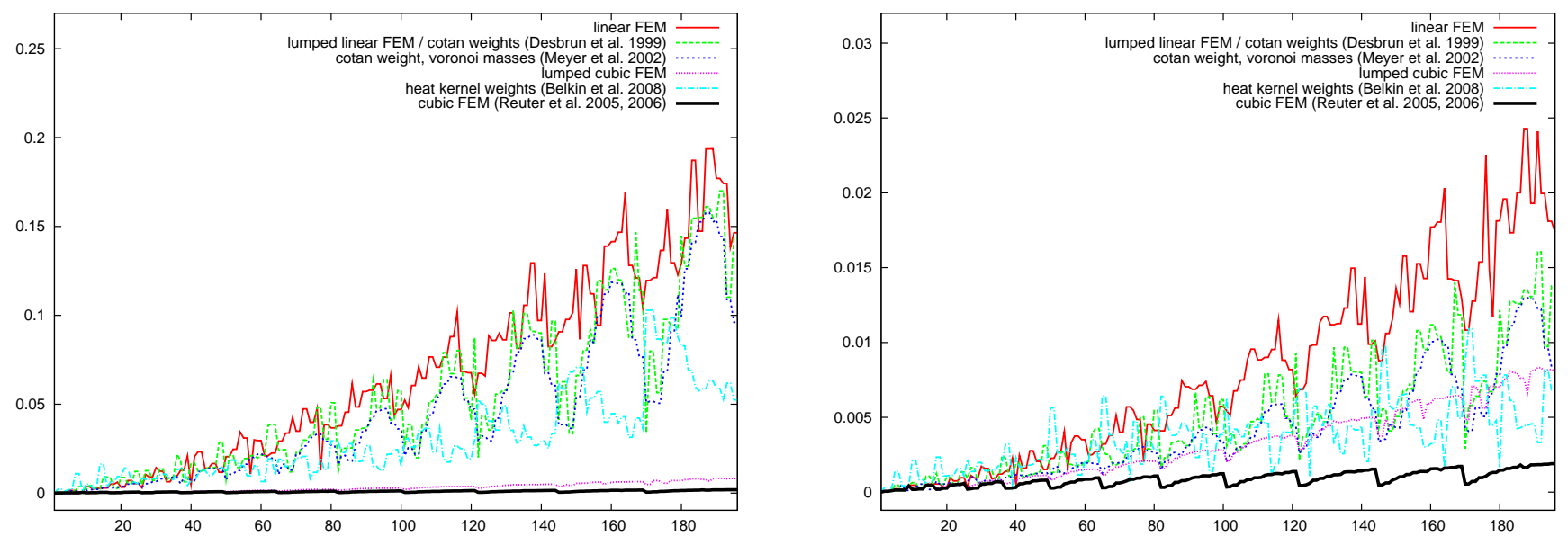

Figure 4: Left: Error of eigenfunctions on the sphere. Right: Comparison with linear methods on the globally refined mesh.

Any function $f$ can be written as a linear combination of all eigenfunctions $f=\sum_{i}^{\infty} c_{i} \psi_{i}$ where the coefficients are $c_{i}=\left\langle f, \psi_{i}\right\rangle=\iint_{M} f \psi_{i} d \sigma$. In the discrete case, we have a given function $f=\sum_{i}^{n} f\left(\mathbf{p}_{i}\right) \varphi_{i}$, where the $\varphi_{i}$ are a basis of functions on the mesh (e.g., the partially linear hat-functions) and $f\left(\mathbf{p}_{i}\right)$ are the values at the vertices. The eigenfunctions $\psi_{j}=\sum_{i}^{n} \psi_{j, i} \varphi_{i}$ are also given by the values at the nodes. Thus, the coefficients $c_{i}$ can be computed

$$
c_{i}=\iint_{M} f \psi_{i} d \sigma=\left(\psi_{i, 1}, \ldots \psi_{i, n}\right) B\left(\begin{array}{c}
f\left(\mathbf{p}_{1}\right) \\
\vdots \\
f\left(\mathbf{p}_{n}\right)
\end{array}\right)
$$

or $\mathbf{c}=Z^{T} B \mathbf{f}$, with $Z$ the matrix of orthonormal eigenvectors. $Z^{T} B$ can be computed once and many functions $\mathbf{f}$ can be projected quickly by a matrix-vector product.

We use the method described above to project a numerically obtained eigenfunction $\tilde{f}$ of the different operators onto a linear approximation of the correct eigenspace, which is known for the rectangular domain. So instead of projecting onto all eigenfunctions (as done in Eq. (7)), we only project $\tilde{f}$ unto the linear approximations of the $n$ eigenfunctions that form a basis of the corresponding correct eigenspace. We can then compute $\sqrt{\iint(\tilde{f}-P(\tilde{f}))^{2} d \sigma}$ to measure the error as the distance between the approximation and its projection. The computations were done for the simple case of a uniform mesh on a flat rectangular domain with 1000 vertices. It can be seen in Figure 3 (left) that the cubic FEM approach performs best, followed by the lumped cubic FEM, linear FEM, Meyer et al. [36] and the lumped linear FEM [16]. The approach by Belkin et al. [6] does not work for eigencomputations on meshes with boundary yet and gives large errors when one tries. It will therefore only be tested for the sphere below.

As the cubic FEM uses a higher degree of freedom for the computations (10 nodes in each triangle instead of just 3 ), it was expected to perform best. Nevertheless, Figure 3 (right) shows, that even after a global mesh refinement the linear methods improve but cannot keep up with the cubic FEM with the same degrees of freedom. Again, the lumped cubic FEM (diagonal mass matrix) performs better than the linear methods, but cannot reach the full cubic approach. In general, on this flat geometry lumping leads to a higher error.

Similar computations were performed on the curved geometry of the unit sphere. The used mesh is very regular and has approximately 1000 vertices. As the surface is closed, no boundary condition needs to be applied. The exact solutions on the sphere are given by the spherical harmonics of degree $l$ and order $m$ :

$$
Y_{l}^{m}(\theta, \varphi)=N e^{i m \varphi} P_{l}^{m}(\cos \theta)
$$

where $N$ is a normalization constant and $P_{l}^{m}$ is an associated Legendre function. Figure 4 (left) shows the performance of the different methods on the regular mesh. All linear methods were also computed on the globally refined mesh and compared to the cubic FEM cases, see Figure 4 (right). All the linear methods perform much better on the sphere than on the bounded rectangular domain (especially after mesh refinement; please note the different scales of the plots). Again the cubic FEM approach performs best, followed by the lumped cubic FEM. Belkin et al. [6] outperform all linear approaches for the higher eigenfunctions and even the lumped cubic FEM after mesh refinement. On this curved geometry lumping the linear FEM (i.e. a diagonal mass matrix) leads to more accurate results. This is not true for the cubic FEM case, where the lumped results are still less accurate than the full cubic approach. The oscillating behavior of the error is due to the fact that the sphere has very high dimensional eigenspaces. The first few eigenfunctions of a new eigenspace are very accurate before the error increases. This can also be observed when looking at the corresponding eigenvalues.

\section{Shape segmentation by nodal domains}

In the literature, there is a growing interest in techniques that analyze a given shape by studying the properties of real-valued functions $f$ defined on the shape itself $[8,9]$. Laplacian eigenfunctions yield a library of real-valued functions that are in- 


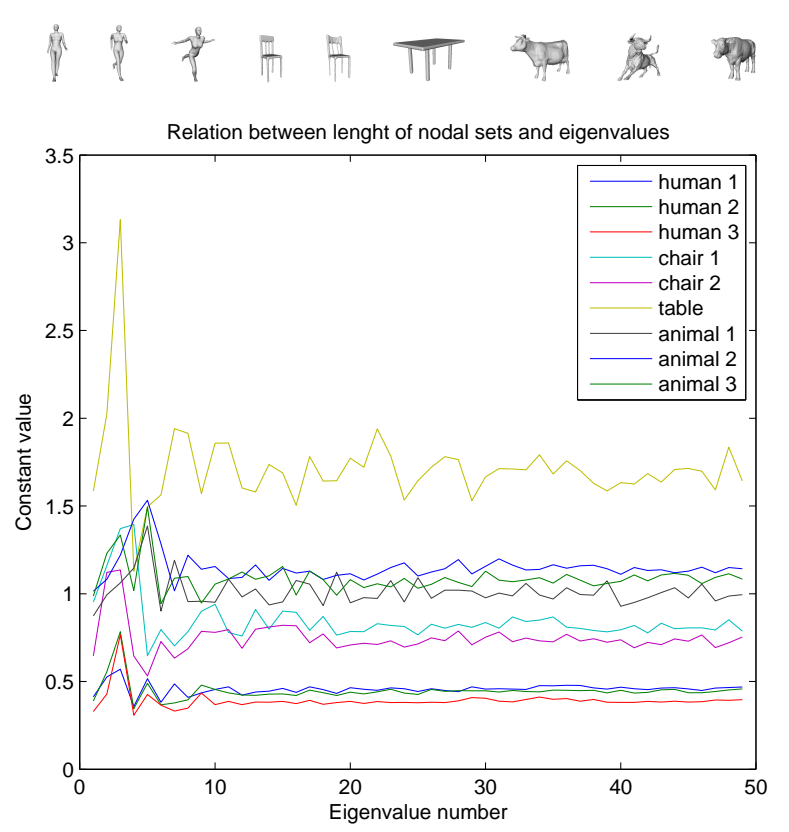

Figure 5: Relation between frequency and nodal sets length.

trinsic to the shape, orthogonal and ordered according to their frequencies. In this paper, we use the nodal sets and nodal domains of the eigenfunctions to derive a set of shape segmentations. According to the correctness in Section 3, the cubic FEM approach is used for the computation of the eigenfunctions.

\subsection{Nodal sets and domains}

The nodal sets $\Gamma_{i}$ are the zero sets of the eigenfunctions of the Laplacian operator on a Riemannian manifold, i.e., $\Gamma_{i}:=f_{i}^{-1}(0)$. A nodal domain is a connected component of the complement of the nodal sets. For $n$-dimensional Riemannian manifolds, each nodal set is a smooth hypersurface and a singular part of dimension less or equal than $n-2$. For surfaces, the nodal sets consist of smooth arcs, called nodal lines, and singular points where these arcs meet. Moreover, where the arcs meet in a singular point, they form an equiangular configuration [4, 12].

Theoretical results on the invariance of the nodal domains of the Laplacian operator with respect to geometric properties have been studied for the torus [32] and the sphere [21] in $\mathbb{R}^{3}$. Lower and upper bounds of the length of the nodal lines have been studied in differential geometry [18, 29, 37]. In general, the measure of the whole nodal set over compact Riemannian surfaces depends on the eigenvalue and is controlled by the geometry of the manifold (i.e., area and curvature). Denoting $\mathcal{L}\left(\Gamma_{i}\right)$ the length of the of the $i^{\text {th }}$ nodal set, the following relation holds: $C_{1} \lambda^{\frac{1}{2}}<\mathcal{L}\left(\Gamma_{i}\right) \leq C_{2} \lambda^{\frac{3}{4}}$, where $C_{1}$ and $C_{2}$ are two constants that exclusively depend on the geometry of the manifold. Figure 5 shows the behavior of the value $\frac{\mathcal{L}\left(\Gamma_{i}\right)}{\lambda^{\frac{1}{2}}}$ on a set of models. As stated in [53], the approximation of the constant becomes more precise as far as the eigenvalue $\lambda_{i}$ increases. Note, that similar shapes have similar curves. As stated by Courant's nodal domain theorem [13], the number of nodal domains of

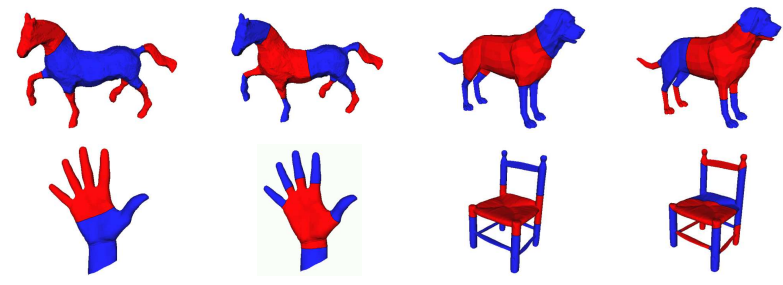

Figure 8: Segmentations induced by the nodal domains of some eigenfunctions selected among the first 15 eigenfunctions.

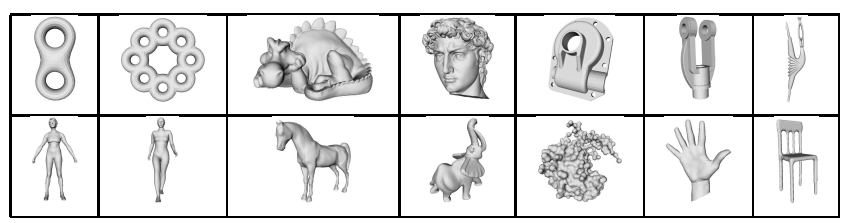

Figure 9: Representative models of our dataset.

the $i^{\text {th }}$ eigenfunction is less than or equal to $i$ and the number of nodal domains of the second eigenfunction is always two [12]. In the discrete case, our experiments confirm that the number $k$ of nodal domains of the $i^{\text {th }}$ eigenfunction respects its theoretical upper bound, i.e., $k \leq i$. Actually, $k$ appears to be considerably smaller than its bound. This can be seen in the diagrams of Figure 6 , which show the growth of the number of nodal domains on four different models. Note, that the number of nodal domains is stable with respect to the different FEM discretizations (lumped, linear, cubic) of the Laplace-Beltrami operator.

\subsection{Nodal sets for shape segmentation}

For each eigenfunction $f_{i}$, the nodal sets decompose a surface into regions where $f_{i}$ has constant sign. In other words, each eigenfunction induces a shape segmentation, with segments corresponding to regions of positive or negative values. The use of nodal sets and nodal domains to segment 3D shapes was addressed in [33]. The first $k$ Laplacian eigenfunctions, ordered according to increasing frequencies, provide a family of shape segmentations, each capturing different shape properties (see Figure 7 and Figure 8).

To evaluate the quality of this set of segmentations, we follow the guidelines given in [2]. The criteria are type and correctness of the segmentation; quality of boundaries; definition of a multi-scale segmentation; invariance to pose; sensitivity to noise and tessellation; computational complexity; and control parameters. To discuss these criteria, we built a set of closed triangle meshes, including surfaces with different shape characteristics (e.g., articulations, complexity, smoothness). The models range from mathematical objects such as tori, to manufactured objects, to animals and humans in different poses The models were collected from several web repositories; many of them are taken from the SHREC 2007 benchmark [23]. Representative models can be seen in Figure 9.

Type and correctness of segmentation. The nodal domains related to the first eigenfunctions subdivide the input surface into patches which have almost the same weight, measured as the 

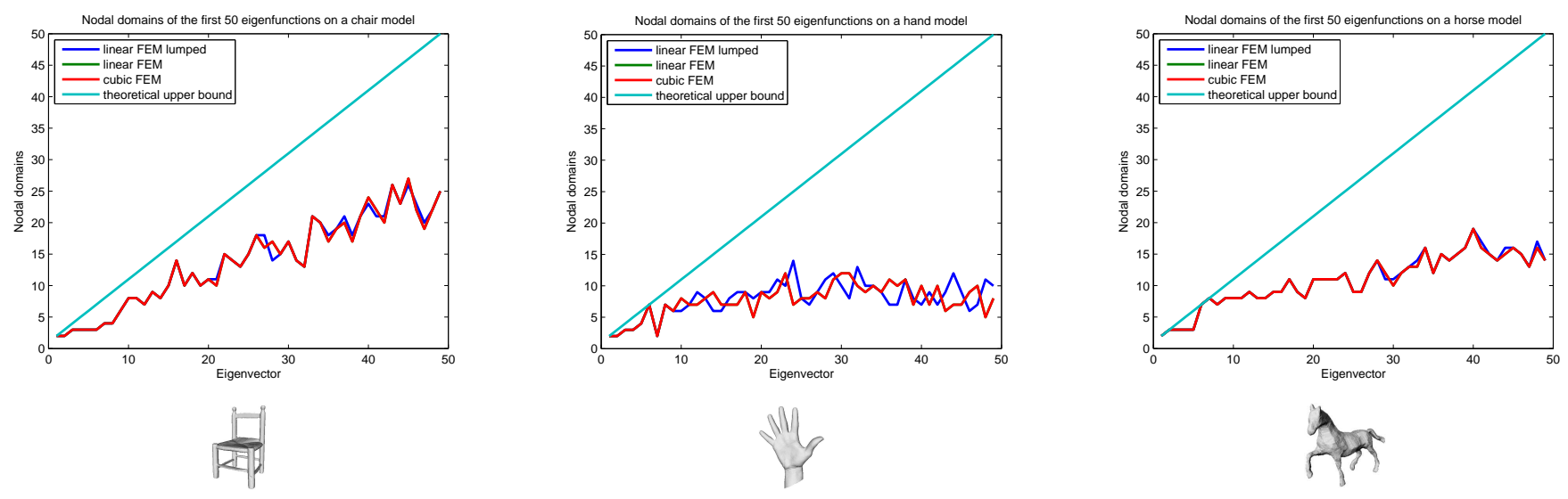

Figure 6: Number of nodal domains in order of increasing eigenvalues.
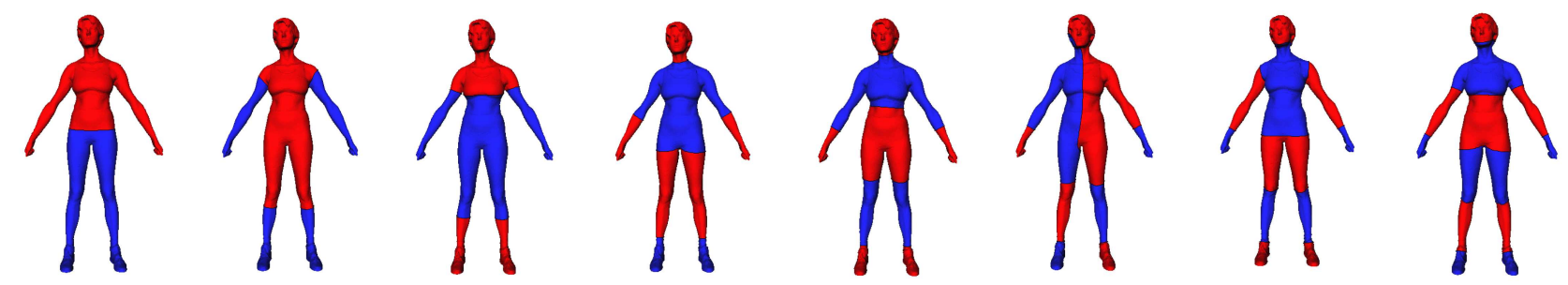

Figure 7: Segmentations induced by the nodal domains of some eigenfunctions selected among the first 15 eigenfunctions (in order of increasing eigenvalues). Blue regions correspond to regions where the eigenfunctions have negative values, while red regions correspond to positive values.
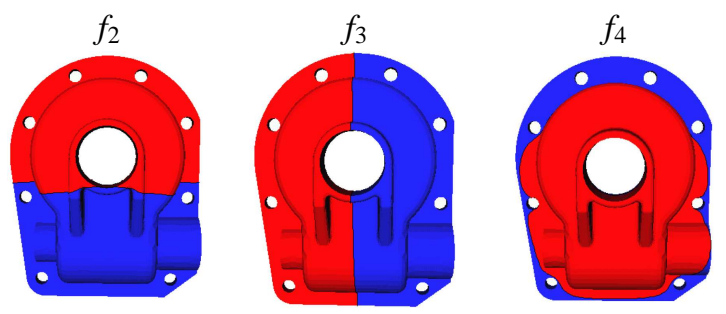

Figure 10: The 2nd, 3rd, 4th eigenfunctions (cubic FEM discretization). The nodal sets of these three eigenfunctions are almost orthogonal.

sum of the edge weights associated with the 1-star of each vertex. In this case, the nodal sets often identify privileged directions, related to the symmetries of the objects (see also [39]). Note, for example, the directions identified by the nodal sets in Figure 10 and 14. For articulated objects, the first eigenvectors define patches that are able to identify surface protrusions, and are often well aligned with perceptual features. In Figure 7 some segmentations of a human model induced by the nodal domains of different eigenfunctions are shown, chosen among the first 15 in the spectrum. At different scales, the segmentations capture the symmetry of the shape, the arms, legs, hands and feet of the model. Other examples are given in Figure 8.

By increasing magnitudes of the eigenvalues, the nodal domains of the corresponding eigenfunctions become smaller disk-like patches, that often spread out almost equally across the shape. The number of patches depends on the surface shape and magnitude of the corresponding eigenvalue; this number is always bounded, as stated in Section 4.1.
Boundaries. Since each eigenfunction discretizes a $C^{2}$ function, we get that the nodal sets $\Gamma_{i}:=f_{i}^{-1}(0)$ identify smooth boundaries of the corresponding nodal domains. The length of the boundaries is related to the frequency of the eigenfunction, as discussed in Section 4.1.

Robustness with isometric transformations and shape pose. As the Laplace-Beltrami operator is isometry invariant, its eigenfunctions do not depend on the embedding but only on the geodesic distances among the points on the surface (the Riemannian metric). Therefore, the segmentations with nodal domains are insensitive to pose changes that barely affect geodesic distances. This is the case for the near-isometric female models in Figure 11. However, if larger non-isometric deformations are involved, it may happen that the nodal domains of some eigenfunctions have different shapes on similar models; e.g. compare the female with the male model in Figure 11.

Multi-scale/hierarchical segmentation. We have seen that the decomposition into nodal domains naturally defines a family of shape segmentations. Note, that the nodal domains do not provide a hierarchical segmentation (i.e., a refined segmentation is a sub-segmentation of a coarse one); the number of nodal domains of the eigenfunction $f_{i}$ is usually higher than that of $f_{j}, i>j$, but an inclusion relation of these sets is not guaranteed. What is relevant to our purposes is that the set of the first eigenfunctions are able to capture a rich set of intrinsic properties, according to different frequencies. Even though not all the segmentations induced by the nodal domains are aligned with the human perception, i.e., not all of them reflect an intuitive 


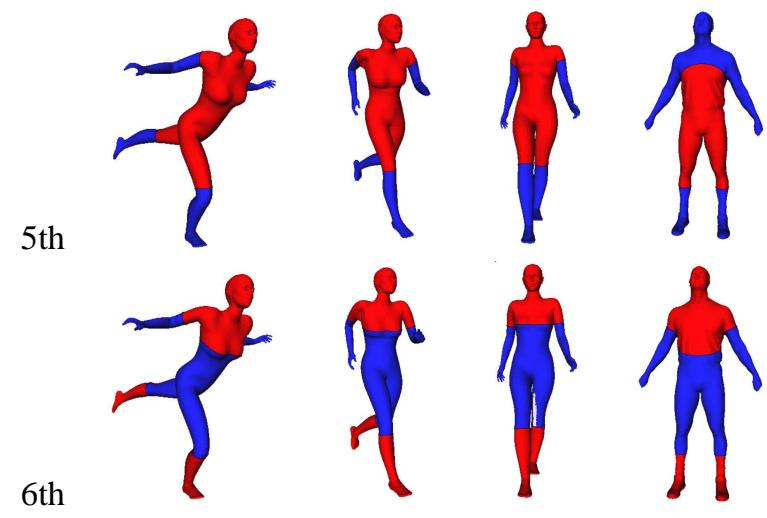

Figure 11: Segmentation of near-isometric and similar models.

segmentation, a satisfactory set of semantically meaningful features is comprised in the segmentations induced by the first part of the spectrum. Because each feature may be captured by a different eigenfunction, the desired segmentation comes from the composition of different segmentations. This concept is in line with the ideas in [3], where different segmentation algorithms are used in parallel, and the desired features are taken from all their results by means of a user-friendly tool. An example of derived segmentation using the Laplacian nodal domains is shown in Figure 12, where the segmentations induced by three eigenfunctions are composed to define two meaningful segmentations, according to the user intent. The head comes from the 8th eigenfunction (it could also have been taken from the 12th eigenfunction, if the neck was to be excluded) as well as the legs, while the arms are those identified either by the 5 th eigenfunction (in case the hands are not required to be segmented) or the 12th eigenfunction (in case the desired level of detail includes the segmentation of the hands). Similar results can be obtained for other objects, as those in Figure 8; for example, we could compose the segmentations induced by the first part of the spectrum of the chair, to separate the seat from the rear part and the legs, or even to separate the singular tubular components.

Sensitivity to noise and tessellation. Figure 13 shows the nodal domains of four eigenfunctions of a smooth and a noisy version of the same shapes, obtained by adding uniform Gaussian noise. The nodal domains, and hence the segmentation, appear to be quite stable with respect to the noise. However, we observe that the connectivity of the zero sets may slightly differ between the original and the noisy model, as it happens for the 4th eigenfunction of the woman model in Figure 13. Similarly, the nodal domains of the first eigenfunctions are quite stable with respect to different samplings of the shape, as shown in Figure 14. As expected, we noticed that the first part of the spectrum is less sensitive to noise and sampling density than the part corresponding to higher eigenvalues.

Asymptotic complexity. Once an eigenfunction has been computed, the extraction of the corresponding nodal sets is linear in the number of intersected edges; therefore, $O(n)$ in the worst case. In order to recognize the regions where the sign of the

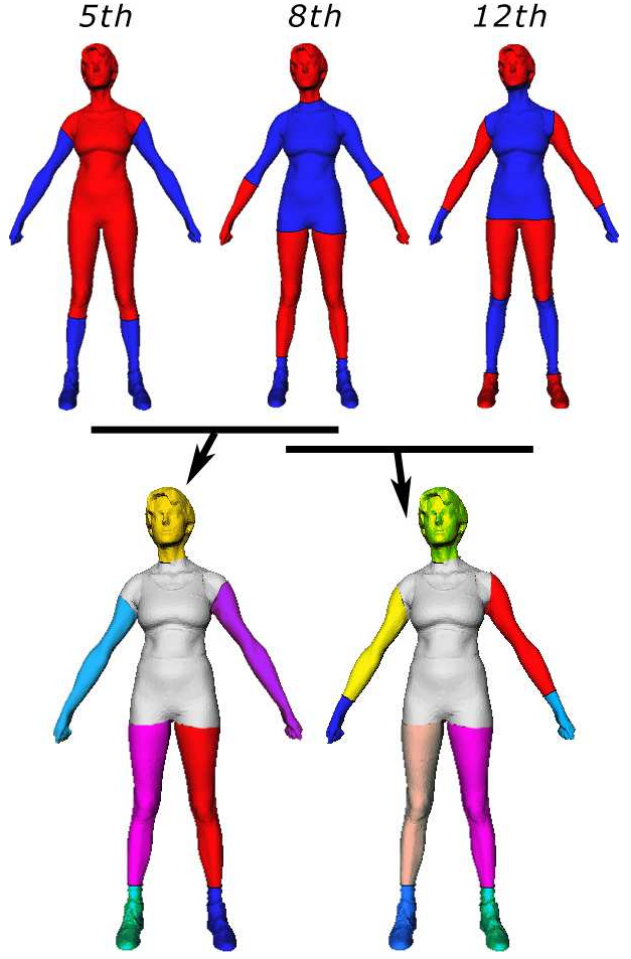

Figure 12: Derived segmentations.

Laplacian eigenfunction is constant, the triangle mesh may be visited only once, hence the extraction of the nodal domains is linear in the number of triangles.

Control parameters. The parameter to define the set of segmentations is given by the number $k$ of eigenfunctions taken into account. In our experiments, we visually examined the segmentations and found that, in general, a low number (e.g., 20) of eigenfunctions is sufficient to extract a good set of perceptually relevant shape features. Note, that it is possible to adopt different strategies to select a proper range of eigenfunctions [17, 34].

\section{Concluding remarks}

In this paper, we have analyzed the correctness of the Laplacian eigenfunctions of different discretizations of the LaplaceBeltrami operator. Then, we have selected the FEM operators for eigenfunctions computation, and derived a set of segmentations from the nodal domains of the eigenfunctions in the first part of the Laplacian spectrum.

From the analysis of the properties of these segmentations, we can derive some ideas about their application for different purposes. A first application could be shape parametrization, because the nodal domains segment the shape into primitives, which define a chart decomposition of the mesh. The nodal sets provide smooth cuts and a low number of patches (see Figure 15), which are useful in case of surfaces with a high genus. If the nodal domains have 0-genus, each chart can be parameterized using an extension of the barycentric coordinates [41, 61]. Moreover, the Laplacian eigenfunction can also be used to define cut-graphs of arbitrary 3D shapes [42, 55]. 


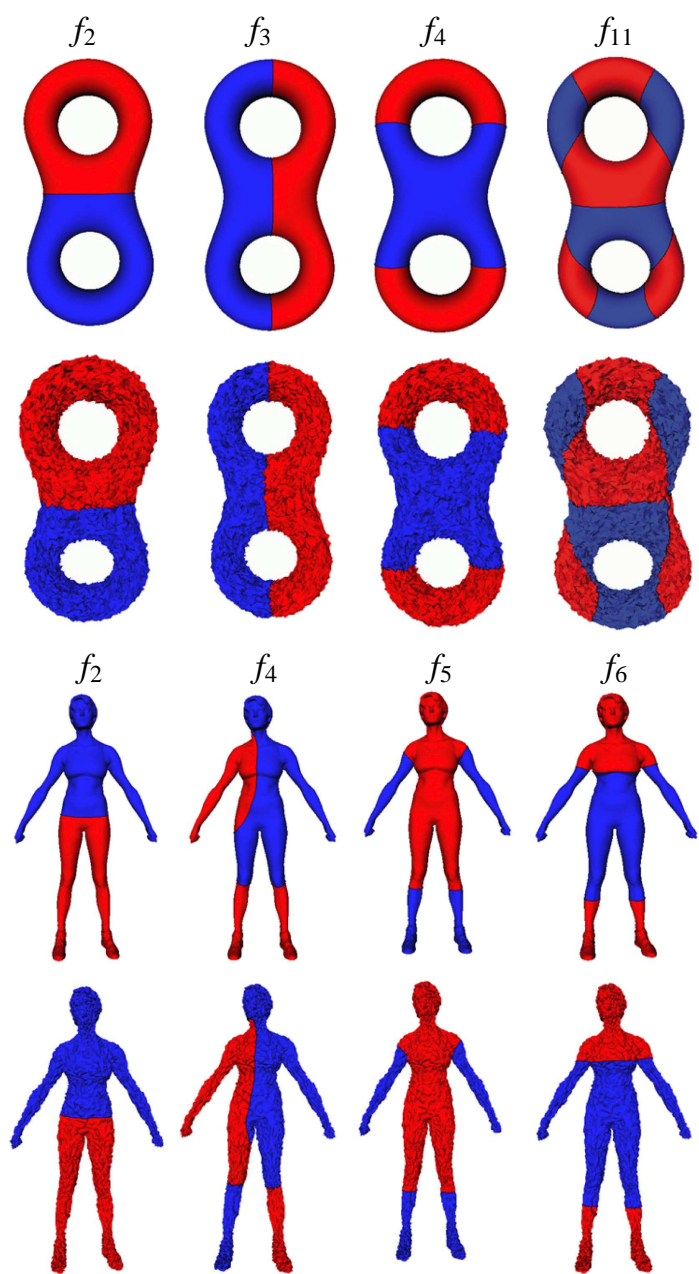

Figure 13: The nodal domains of the 2nd, 3rd, 4th and 11th eigenfunctions (linear FEM) on a bitorus and a woman model (top) and a noisy version of the same models (bottom), obtained by adding Gaussian noise (width $\sigma=2 \%$ of the radius of the enclosing sphere) to surface vertices.

A second possibility is the definition of a multi-scale signature for 3D shape description and comparison. We are currently investigating the use of the sequence of the decomposition graphs that encode the adjacency among the nodal domains as a multi-scale descriptor. More precisely, the descriptor is the set $G=\bigcup_{i=2}^{n} G_{i}$, where $G_{i}=\left(V_{i}, E_{i}\right)$ is the decomposition graph derived from the $i^{\text {th }}$ eigenfunction, the nodes in $V_{i}$ correspond to the nodal domains of the eigenfunction, and the edges in $E_{i}$ mimic the adjacency relationships among the nodal domains. An example of this representation using the first nine eigenfunctions is shown for a human model in Figure 16. The advantage of this descriptor with respect to others that could be derived from the Laplacian eigenfunctions, as those related to critical points, comes from the experimental observation that the number of nodal domains increases less rapidly than the number of critical points, see Figure 17. Hence, while shape descriptors based on critical points such as Morse and MorseSmale complexes, contour trees, Reeb graphs, size theory and persistent homology [9], may become complex when the order of the eigenfunctions increases, the proposed descriptor is ex-

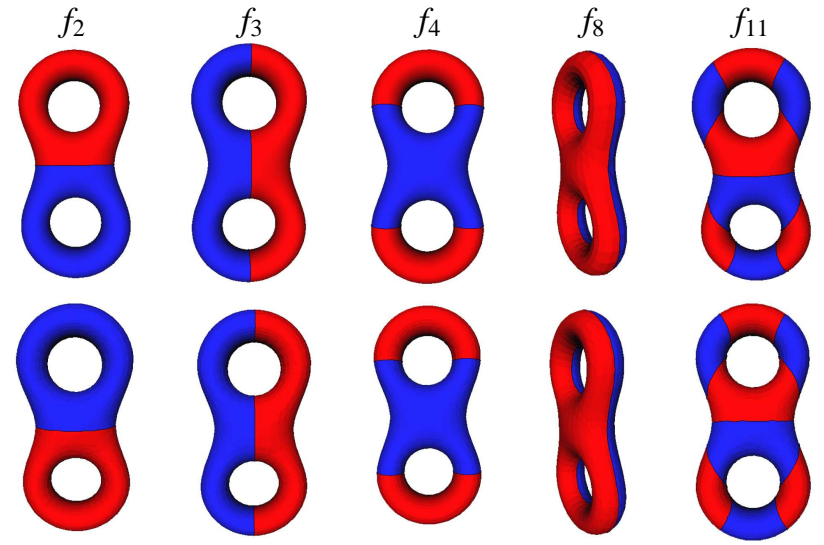

Figure 14: The 2nd, 3rd, 4th, 8th and 11th eigenfunctions (linear FEM discretization) and their nodal domains on a bitorus with $13 \mathrm{~K}$ vertices (top) and a simplified version of the same model with 1500 vertices (bottom).

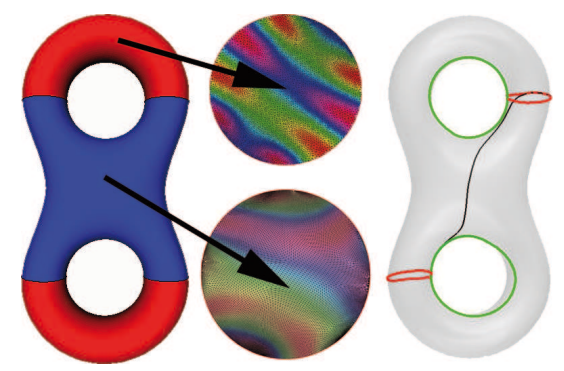

Figure 15: (Left) Surface segmentation into three nodal domains and corresponding embeddings on the parameterization domain. (Right) Cut-graph.

pected to remain more concise.

An important issue for further research is the development of a method for automatically selecting eigenfunctions with desirable segmentation. We are currently investigating on that with the aim of finding a set of eigenfunctions that contain in themselves the most relevant shape properties.

\section{Acknowledgments}

The authors would like to thank Bianca Falcidieno for her valuable support. Special thanks are also given to the reviewers for their comments and suggestions. This work has been partially supported by the FOCUS K3D Coordination Action, EU Contract ICT-2007.4.2 contract number 214993 and the Italian National Project SHALOM funded by the Italian Ministry of Research under contract number RBIN04HWR8. Moreover, this work was partially funded by a Humboldt Foundation postdoctoral fellowship to the first author. Models are courtesy of the AIM@SHAPE repository.

\section{References}

[1] ARPACK The Arnoldi Package http://www.caam.rice.edu/software/ARPACK/.

[2] M. Attene, S. Katz, M., G. Patanè, M. Spagnuolo, and A. Tal. Mesh segmentation - a comparative study. In Proc. of Shape Modeling Internations, page 7,2006 

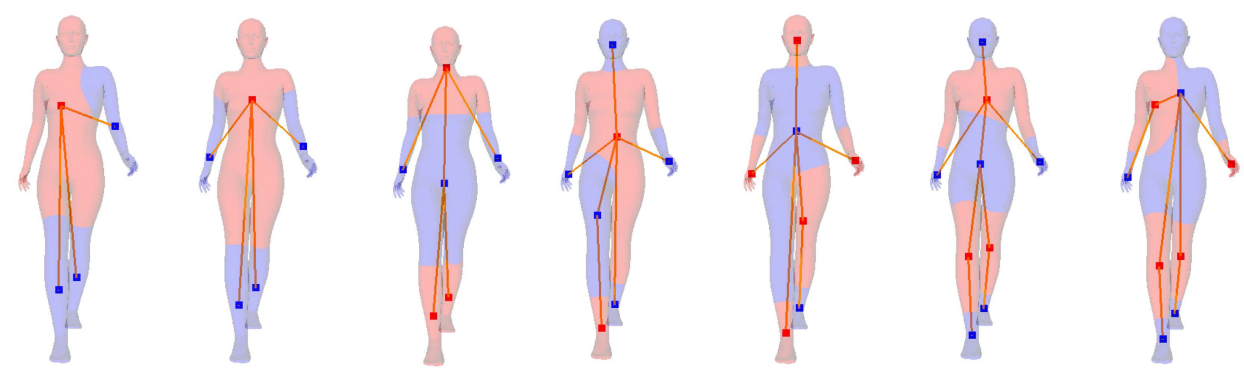

Figure 16: Sequence of decomposition graphs of a human model.

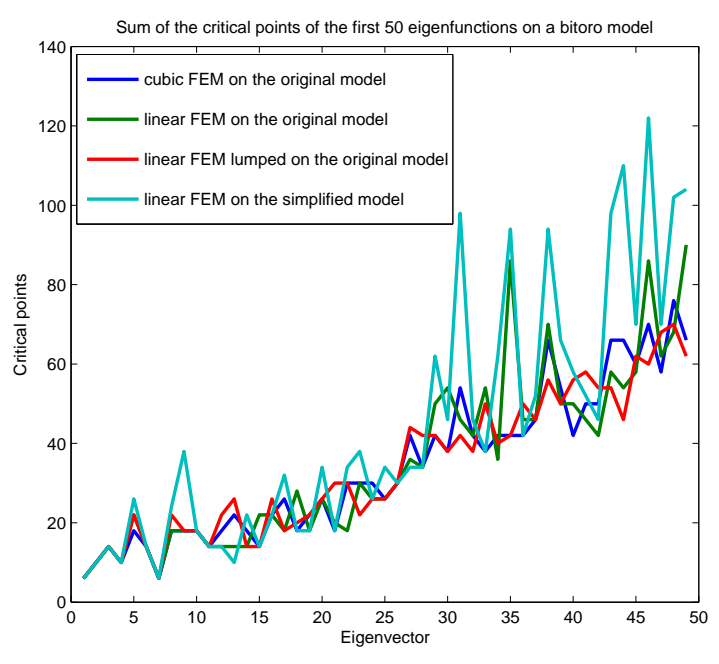

(a)

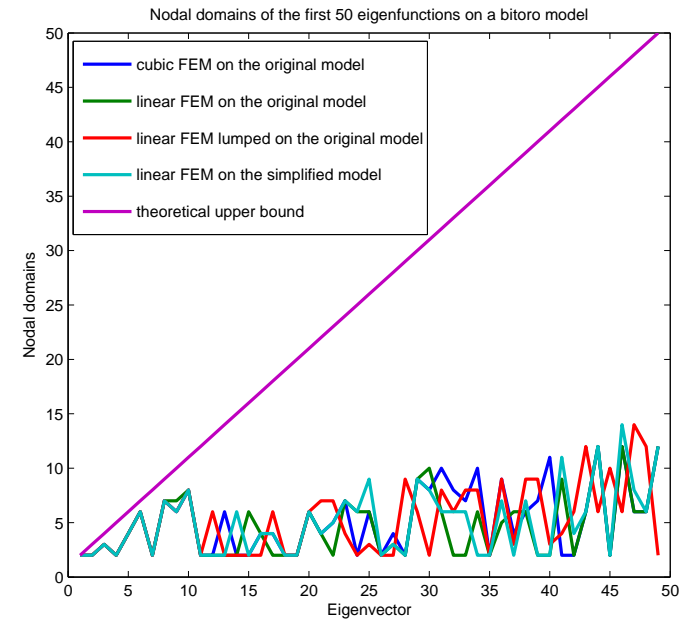

(b)

Figure 17: (a) Progress of the number of critical points and (b) nodal domains of the bitorus model with respect to the different eigenfunctions.

[3] M. Attene, F. Robbiano, M. Spagnuolo, and B. Falcidieno. Semantic annotation of 3D surface meshes based on feature characterization. Proc. Semantic and Digital Media Technologies, 4816:126-139, 2007.

[4] C. Bar. On nodal sets for Dirac and Laplace operators. Communications in Mathematical Physics, 188:709-721, 1997.

[5] M. Belkin and P. Niyogi. Laplacian eigenmaps for dimensionality reduction and data representation. Neural Computations, 15:1373-1396, 2003.

[6] M. Belkin, J. Sun, and Y. Wang. Discrete Laplace operator on meshed surfaces. In Symposium on Computational Geometry, pages 278-287, 2008.

[7] M. Berger, P. Gauduchon, and E. Mazet. Le Spectre d'une Variété Riemannienne. Springer Verlag, 1971.

[8] S. Biasotti, B. Falcidieno, P. Frosini, D. Giorgi, C. Landi, G. Patanè, and M. Spagnuolo. 3D shape description and matching based on properties of real functions. In Eurographics 2007 Tutorial Proc., pages 949-998, 2007.

[9] S. Biasotti, L. De Floriani, B. Falcidieno, P. Frosini, D. Giorgi, C. Landi, L. Papaleo, and M. Spagnuolo. Describing shapes by geometricaltopological properties of real functions. ACM Computing Surveys, 40(4):1-87, 2008.

[10] I. Biederman. Recognition-by-components: A theory of human image understanding. Psychological Review, 94(2):115-147, 1987.

[11] I. Chavel. Eigenvalues in Riemannian Geometry. Academic Press, 1984.

[12] S.-Y. Cheng. Eigenfunctions and nodal sets. Comment. Math. Helvetici, 51:43-55, 1976.

[13] R. Courant and D. Hilbert. Methods of Mathematical Physics, volume 1. Interscience, New York, 1953.

[14] J. W. Demmel, S. C. Eisenstat, J. R. Gilbert, X. S. Li, and J. W. H. Liu. A supernodal approach to sparse partial pivoting. SIAM Journal on Matrix Analysis and Applications, 20(3):720-755, 1999.

[15] M. Desbrun, E. Kanzo, and Y. Tong. Discrete differential forms for com- putational modeling. In ACM Siggraph '05 Course notes on Discrete Differential Geometry, Chapter 7, 2005.

[16] M. Desbrun, M. Meyer, P. Schröder, and A.H. Barr. Implicit fairing of irregular meshes using diffusion and curvature flow. In Proc. ACM Siggraph '99, pages 317-324, 1999.

[17] S. Dong, P.-T. Bremer, M. Garland, and V. Pascucci. Spectral surface quadrangulation. In Proc. ACM Siggraph, pages 1057-1066, 2006.

[18] H. Donnelly and C. Fefferman. Nodal sets for eigenfunctions of the Laplacian on surfaces. J. Amer. Math. Soc., 3:333-353, 1990.

[19] R. Dyer, H. Zhang, T. Möller, and A. Clements. An investigation of the spectral robustness of mesh Laplacians. Technical Report 2007-17, SFU CS School, 2007.

[20] G. Dziuk. Finite elements for the Beltrami operator on arbitrary surfaces. In S. Hildebrandt and R. Leis, editors, Partial differential equations and calculus of variations, pages 142-155. 1988.

[21] A. Eremenko, D. Jakobson, and N. Nadirashvili. On nodal sets and nodal domains on $S^{2}$ and $R^{2}$. Annales de l'Istitut Fourier, 57(7):2345-2360, 2007.

[22] M. S. Floater and K. Hormann. Surface parameterization: a tutorial and survey. In Advances in Multiresolution for Geometric Modelling, pages 157-186. Springer Verlag, 2005.

[23] D. Giorgi, S. Biasotti, and L. Paraboschi. Watertight models track. Technical Report 09, IMATI, Genova, 2007.

[24] F. M. Gomes and D. C. Sorensen. ARPACK++: A C++ implementation of ARPACK eigenvalue package.

[25] C. Gotsman, X. Gu, and A. Sheffer. Fundamentals of spherical parametrization for 3D meshes. ACM Trans. Graph., 3(22):358-363, 2003.

[26] DD Hoffman and WA Richards. Parts of recognition. Cognition, 18(13):65-96, 1984.

[27] V. Jain and H. Zhang. Robust 3D shape correspondence in the spectral 
domain. In Proc. Shape Modeling International 2006, pages 118-129, 2006.

[28] V. Jain and H. Zhang. A spectral approach to shape-based retrieval of articulated 3D models. Coumputer Aided Design, 39(5):398-407, 2007.

[29] D. Jakobson, N. Nadirashvili, and J. Toth. Geometric properties of eigenfunctions. Russian Math. Surveys, 56:67-88, 1991.

[30] Z. Karni and C. Gotsman. Spectral compression of mesh geometry. In ACM Siggraph, pages 279-286, 2000.

[31] S. Katz, G. Leifman, and A. Tal. Mesh segmentation using feature point and core extraction. The Visual Computer, 21:649-658, 2005.

[32] J. P. Keating, F. Mezzadri, and A. G. Monastra. Nodal domain distributions for quantum maps. J. Phys. A: Math. Gen., 36(3):53-59, 2003.

[33] B. Lévy. Laplace-Beltrami eigenfunctions: towards an algorithm that understands geometry. In Proc. of Shape Modeling and Applications, page 13, 2006

[34] B. Lévy and B. Vallet. Spectral geometry processing with manifold harmonics. Computer Graphics Forum, 2(27), 2008.

[35] R. Liu and H. Zhang. Mesh segmentation via spectral embedding and contour analysis. Computer Graphics Forum, 26(3):385-394, 2007.

[36] M. Meyer, M. Desbrun, P. Schröder, and A.H. Barr. Discrete differentialgeometry operators for triangulated 2-manifolds. In Proc. VisMath 2002, 2002.

[37] N. S. Nadirashvili. Metric properties of eigenfunctions of the Laplace operator on manifolds. Annales de l'Istitut Fourier, 41(1):259-265, 1991.

[38] M. Niethammer, M. Reuter, F.-E. Wolter, S. Bouix, N. Peinecke, M. S. Koo, and M. E. Shenton. Global medical shape analysis using the Laplace-Beltrami spectrum. In MICCAI Conference, pages 850-857. Springer, 2007.

[39] M. Ovsjanikov, J. Sun, and L. Guibas. Global intrinsic symmetries of shapes. In Eurographics Symposium on Geometry Processing (SGP), 2008.

[40] B. N. Parlett. The Symmetric Eigenvalue Problem. Prentice-Hall, Englewood Cliffs, 1980.

[41] G. Patanè, M. Spagnuolo, and B. Falcidieno. Para-graph: graph-based parameterization of triangle meshes with arbitrary genus. Computer Graphics Forum, 23(4):783-797, 2004.

[42] G. Patanè, M. Spagnuolo, and B. Falcidieno. Families of cut-graphs for bordered meshes with arbitrary genus. Graphical Models, 69(2):119-138, 2007.

[43] G. Patanè, M. Spagnuolo, and B. Falcidieno. Topological generators and cut-graphs of arbitrary triangle meshes. In Proc. of Shape Modeling and Applications 2007, pages 113-122, 2007.

[44] N. Peinecke, F.-E. Wolter, and M. Reuter. Laplace spectra as fingerprints for image recognition. Computer Aided Design, 39(6):460-476, 2007.

[45] U. Pinkall and K. Polthier. Computing discrete minimal surfaces and their conjugates. Experimental Mathematics, 1(2), 1993.

[46] M. Reuter. Laplace Spectra for Shape Recognition. Books on Demand, ISBN 3-8334-5071-1, 2006.

[47] M. Reuter, M. Niethammer, F.-E. Wolter, S. Bouix, and M. E. Shenton. Global medical shape analysis using the volumetric Laplace spectrum. In IEEE Proc. Cyberworlds 2007, pages 417-426, 2007.

[48] M. Reuter, F.-E. Wolter, and N. Peinecke. Laplace-spectra as fingerprints for shape matching. In Proc. of the ACM Symp. on Solid and Physical Modeling, pages 101-106, 2005.

[49] M. Reuter, F.-E. Wolter, and N. Peinecke. Laplace-Beltrami spectra as Shape-DNA of surfaces and solids. Computer Aided Design, 38(4):342366, 2006

[50] M. Reuter, F.-E. Wolter, M. Shenton, and M. Niethammer. LaplaceBeltrami eigenvalues and topological features of eigenfunctions for statistical shape analysis. Computer Aided Design, 2009.

[51] S. Rosenberg. The Laplacian on a Riemannian Manifold. Cambridge University Press, 1997.

[52] R. M. Rustamov. Laplace-beltrami eigenfunctions for deformation invariant shape representation. In Symposium on Geometry Processing, pages 225-233, 2007.

[53] A. Savo. Lower bounds for the nodal length of eigenfunctions of the Laplacian. Annals of Global Analysis and Geometry, 19(2):133-151, 2001.

[54] A. Shamir. Segmentation and shape extraction of 3D boundary meshes. In Eurographics STAR Report, 2006.

[55] D. Steiner and A. Fischer. Cutting 3D freeform objects with genus-n into single boundary surfaces using topological graphs. In Proc. of the Symp. on Solid Modeling and Applications, pages 336-343, 2002.

[56] G. Strang and G. J. Fix. An Analysis of the Finite Element Method. Prentice-Hall, 1973.

[57] G. Taubin. A signal processing approach to fair surface design. In $A C M$ Siggraph'95, pages 351-358, 1995.

[58] M. Wardetzky, M. Bergou, D. Harmon, D. Zorin, and E. Grinspun. Discrete quadratic curvature energies. Computer Aided Geometric Design, 8(21):398-407, 2007.

[59] M. Wardetzky, S. Mathur, F. Kalberer, and E. Grinspun. Discrete Laplace operators: No free lunch. In Proc. Symp. Geom. Proc. 2007, 2007.

[60] G. Xu. Discrete Laplace-Beltrami operators and their convergence. Computer Aided Geometric Design, 21:767-784, 2004.

[61] E. Zhang, K. Mischaikow, and G. Turk. Feature-based surface parameterization and texture mapping. ACM Trans. Graph., 24(1):1-27, 2005.

[62] H. Zhang, O. van Kaick, and R. Dyer. Spectral methods for mesh processing and analysis. In Eurographics STAR Report, pages 1-22, 2007. 